\title{
Lacrimal Gland Tumours in Dogs: A Histological and Immunohistochemical Study
}

L.Nordio*N.Perelli*D.MultaritC.Giudice*

Introduction: Lacrimal gland tumours (LGTs) are well-known neoplasms affecting both the nictitating membrane and the main lacrimal glands in dogs; however, extensive histological and immunohistochemical studies are currently lacking. In recent years an increasing number of LGTs with a complex (i.e. epithelial and myoepithelial) phenotype have been recorded in our routine histopathology service. The aim of the present study was to review histological and immunohistochemical features of LGTs in dogs.

Material and Methods: Nictitating membrane (NM) and main lacrimal gland (LG) biopsy samples from the departmental archive (2010-2016) were reviewed. $\mathrm{HE}$ sections were re-evaluated by light microscopy. Since a specific classification for LGTs is currently lacking, the classification proposed by Goldschmidt (2011) for mammary gland tumours was applied. Immunohistochemistry for pancytokeratin (CK AE1/AE3), CK14, a-smooth muscle actin, calponin and p63 was performed.

Results: One-hundred and sixty-one NM and LG specimens were reviewed: 106 neoplastic lesions were diagnosed, 28 were epithelial gland tumours (25 NM; three LG). Twenty-four of 28 were carcinomas: seven complex, five solid, two simple tubular, two tubulopapillary, two comedocarcinoma, one ductal, four carcinoma and malignant myoepithelioma, one malignant myoepithelioma. Four were benign tumours: two complex adenomas, two multilobular adenomas. CKAE1/AE3 labelled tubular cells, while CK14, $\alpha$-SMA, calponin and p63 labelled myoepithelial cells, when present.

Conclusions: In the present cohort of cases, NM and LG complex carcinomas and adenomas were highly represented among LGTs. NM and LG carcinomas outnumbered adenomas and were characterized by marked intratumoural variability. A specific classification for lacrimal tumours is needed. 